\title{
BMJ Open Marathon Kids UK: study design and protocol for a mixed methods evaluation of a school-based running programme
}

\author{
Anna E Chalkley, ${ }^{1}$ Ash C Routen, ${ }^{1}$ Jo P Harris, ${ }^{1}$ Lorraine A Cale, ${ }^{1}$ Trish Gorely, ${ }^{2}$ \\ Lauren B Sherar ${ }^{1}$
}

To cite: Chalkley AE, Routen AC, Harris JP, et al. Marathon Kids UK: study design and protocol for a mixed methods evaluation of a school-based running programme. BMJ Open 2018;8:e022176. doi:10.1136/ bmjopen-2018-022176

- Prepublication history for this paper is available online. To view these files, please visit the journal online (http://dx.doi. org/10.1136/bmjopen-2018022176).

Received 6 February 2018 Revised 17 March 2018 Accepted 6 April 2018
Check for updates

${ }^{1}$ National Centre for Sport and Exercise Medicine, School of Sport, Exercise and Health Sciences, Loughborough University, Loughborough, UK ${ }^{2}$ Department of Nursing, University of the Highlands and Islands, Loughborough, UK

Correspondence to Anna E Chalkley; a.e.chalkley@|boro.ac.uk

\section{ABSTRACT}

Introduction Schools are promising settings for physical activity promotion; however, they are complex and adaptive systems that can influence the quality of programme implementation. This paper presents an evaluation of a school-based running programme (Marathon Kids). The aims of this study are (1) to identify the processes by which schools implement the programme, (2) identify and explain the contextual factors affecting implementation and explications of effectiveness and (3) examine the relationship between the level of implementation and perceived outcomes.

Methods Using a realist evaluation framework, a mixed method single-group before-and-after design, strengthened by multiple interim measurements, will be used. Year 5 (9-10 years old) pupils and their teachers will be recruited from six state-funded primary schools in Leicestershire, UK. Data will be collected once prior to implementation, at five discrete time points during implementation and twice following implementation. A weekly implementation log will also be used. At time point 1 (TP1) (September 2016), data on school environment, teacher and pupil characteristics will be collected. At TP1 and TP6 (July 2017), accelerometry, pupil self-reported physical activity and psychosocial data (eg, social support and intention to be active) will be collected. At TP2, TP3 and TP5 (January, March and June 2017), observations will be conducted. At TP2 and TP5, there will be teacher interviews and pupil focus groups. Follow-up teacher interviews will be conducted at TP7 and TP8 (0ctober 2017 and March 2018) and pupil focus group at TP8. In addition, synthesised member checking will be conducted (June 2018) with a mixed sample of schools.

Ethics and dissemination Ethical approval for this study was obtained through Loughborough University Human Participants Ethics Subcommittee (R16-P032 \& R16-P116). Findings will be disseminated via print, online media and dissemination events as well as practitioner and/or research journals.

\section{INTRODUCTION}

School-based physical activity programmes have rapidly grown in within the public health policy agenda over recent years. ${ }^{1}$ This has predominantly been driven by efforts to address increases in childhood obesity. ${ }^{2}$ Indeed, the recent Childhood Obesity

\section{Strengths and limitations of this study}

This study will result in a rich dataset, reflecting multiple levels, which will expand knowledge on translation of school-based physical activity programmes into practice.

- Data will be collected over a two year period, making it possible to evaluate sustainability of implementation.

- Adopting a realist framework to evaluate implementation, on different levels and using mixed methods, will develop a better understanding of the complexity of implementing physical activity programmes in schools.

- The evaluation will only focus on one year group within the schools, which may limit the applicability of the findings across the primary school setting.

Strategy $^{3}$ outlined ambitions for schools to provide at least $30 \mathrm{~min}$ of moderate to vigorous physical activity (MVPA) a day through active break times, physical education (PE), extracurricular clubs, active lessons and/or other sport and physical activity events. Interestingly, the strategy specifically identified running programmes as a potential approach schools could use to provide additional physical activity time. Intuitively, these programmes offer an attractive prospect for schools due to their simplicity and apparent replicability. They typically consist of pupils regularly walking/running, while in school uniform, along a marked route on the school grounds (field and/or playground) for approximately $15 \mathrm{~min}$.

The combination of additional funding for PE, school sport and physical activity, ${ }^{45}$ government support of running programmes and the 'grass roots' style movement, led by initiatives such as The Daily Mile ${ }^{6}$ has created a 'perfect storm' for schools to address inactivity. ${ }^{7}$ Although these programmes offer opportunities for children to increase their physical activity levels in school, schools are complex social structures with unique 
qualities that can influence the quantity and quality of implementation and do not always allow for programmes to be transferred from one school to another with predictable results. ${ }^{8}$ In order to maximise the potential of running programmes, there is a need to understand how they can best be delivered, and the factors that influence their implementation, in order to ensure they are being used effectively and that all children, including the least active, are engaged.

Achieving optimal implementation is challenging, and researchers have suggested that suboptimal implementation may dilute an intervention's effects and potentially account for small effect sizes in many schoolbased interventions. ${ }^{9-12}$ A programme's lack of success could be attributed to any number of reasons, including poor design, poor or incomplete implementation and/ or failure to reach sufficient numbers of the target audience. ${ }^{13}$ However, only examining programme-related reasons overlooks the potential impact of contextual factors on implementation and subsequently outcomes. Implementation evaluations can describe and explain what was delivered, how implementation was achieved, as well as the contextual factors that might explain intervention outcomes and understanding of intervention theory, thereby narrowing the gap between intervention development and its application in practice. ${ }^{14}$

An increasing emphasis is now placed on measuring implementation, in part because of great variability in programme implementation and policy adoption in school settings. ${ }^{15}$ However, implementation evaluation outside of the USA is infrequent, and there are a limited number of such studies that have been found within a UK context (eg, refs ${ }^{16}$ ). It is likely that differences in educational policies and practices within the UK mean that there are salient issues impacting on implementation processes, relating to national contexts, which may not have been reported elsewhere in the literature. ${ }^{18}$

\section{AIM}

The purpose of this paper is to outline the study design and protocol for an implementation evaluation to examine the implementation of Marathon Kids (MK), a primary school-based running programme.

The objectives of this study are to:

- Identify the processes by which teachers and schools implement the programme.

- Identify and explain the contextual factors most likely to contribute to implementation and explications of effectiveness.

- Examine the relationship between the level of implementation of MK and perceived outcomes.

\section{Programme description}

$\mathrm{MK}^{19}$ gives primary school pupils the opportunity to run or walk the distance of a marathon over a given period (ie, the school year). The programme aims to inspire and empower children of all fitness and ability levels to achieve by using personalised goal setting. It is managed and delivered by the charity Kids Run Free (KRF) who provide opportunities for children to take part in organised running events. The programme encourages pupils to complete laps of a course on the school grounds once or twice a week during lunch break. For every lap completed, pupils are given an elastic band, which are recorded centrally in school via a digital tracking system (DTS). The DTS provides a computerised log of when pupils participate, how may laps each pupil completes and accumulated distance to date. Distance is monitored over time and rewards are given at key milestones, for example, quarter, half, three-quarter and full marathon. MK shares many characteristics with other school-based running programmes such as The Daily Mile ${ }^{6}$ and Golden Mile, ${ }^{20}$ although it differs in its timing (lunch time rather than class time), monitoring (lap bands) and goal setting (reaching the equivalent of a marathon).

A number of tools and resources are provided to schools to support the programme's implementation via the Marathon Champion (a member of school staff who takes responsibility for coordinating MK in the school). On-site support is also provided during a school launch by a member of staff from KRF who: delivers a marathon themed assembly to staff and pupils, demarcates the running route(s), populates the DTS with pupils' details and provides training on how to administer the programme with the Marathon Champion and a selection of peer leaders or Marathon Ambassadors.

During the summer of 2016, KRF partnered with Nike who also supports Marathon Kids US, a comparable charity in terms of vision and mission that works to support sustainable running clubs in the community. Consequently, Kids Marathon rebranded to Marathon Kids UK, and a number of principles of practice were formally adopted, namely the MK's five pillars of goal setting, tracking, role modelling, celebrating and rewards. In addition, pupils are now encouraged to set themselves goals to complete up to four marathons over the course of the school year. Previous research on the initial model of MK provides some foundation for its potential effectiveness. ${ }^{21}$ This evaluation, however, aims to inform a strategic approach to the growth and development of the UK programme as a result of the partnership with Nike, with specific attention being given to how schools can effectively implement the programme to ensure sustained participation.

\section{METHODS}

\section{Evaluation framework}

A realist evaluation framework ${ }^{22}$ will be used to explore the underlying processes of implementation and mechanisms of action and how these vary by contextual characteristics of person (eg, gender) and place (eg, socioeconomic deprivation). Realist evaluation is a member of a family of theory-based evaluation approaches that recognise that for socially dependent programmes there 
are multiple causal mechanisms operating at different levels and emphasises the contextually contingent nature of these mechanisms. ${ }^{23}$ Specifically, this methodological approach involves the formulation, testing and refining of a number of different hypotheses to describe how the underlying mechanisms are expected to operate in different contexts and what outcomes will be generated. This interaction can be expressed as a context+mechanism=outcome or CMO hypothesis and is the analytical basis for realist evaluation. ${ }^{22}$

Realist methods support the development and use of 'middle range theories', which help to generate transferable learning. The term 'middle-range theory' was developed to distinguish grand social theories from the process of incorporating theory and empirical research to explain patterns of social behaviour and outcomes in a particular social setting. ${ }^{24}$ The development and testing of theories (CMO configurations) within realist evaluation is an example of middle-range theory and research. ${ }^{25}$

For understanding the translation into practice of interventions such as MK and for the purpose of this study, realist principles have much to offer in acknowledging the contextual heterogeneity of the different primary schools and facilitating knowledge about implementation processes, longer term sustainability, benefits and potential unintended consequences, as well as the likely effects of interventions for different localities and population subgroups. ${ }^{14}$ This study will therefore use existing theory and the generation and analysis of empirical data to develop theory-based empirical results to inform how, why, for whom and in what circumstances the programme works or not. Furthermore, it will allow the development of a set of portable principles applicable to other running programmes (such as The Daily Mile) and/or schoolbased physical activity programmes.

\section{Study design}

A mixed method, single-group before-and-after design will be used to empirically test the explanatory framework and help understand and explain the processes and context of implementation. The use of mixed methods in implementation research is advocated ${ }^{26}$ and is particularly pertinent where the input of various stakeholders is needed to understand the contextual influences on implementation, thus maximising the likelihood of findings having practical significance. ${ }^{27}$ Data will be collected at multiple time points throughout the 2016/2017 academic year, with follow-up measures planned for the 2017/2018 academic year.

\section{Study population and recruitment}

The study will be conducted within the East Midlands region of the UK. Through a partnership with a teaching school alliance ${ }^{\mathrm{i}}$, six state-funded primary schools from

${ }^{\mathrm{i}}$ Teaching School Alliances are groups of outstanding schools that work with others to provide high-quality training and support for school improvement in their local area. across Leicestershire, which are new to MK, will be recruited. All schools in the Alliance will be invited to participate via a face-to-face head teacher meeting, facilitated by the Alliance, at which they will be asked to provide an initial expression of interest to take part in the study. With over $85 \%$ of the schools in Leicestershire being part of a teaching school alliance ${ }^{\mathrm{ii}}$, it is felt that this approach is likely to provide a representative sample and include schools that would not necessarily have considered engaging in a programme of this nature, and thereby would provide valuable insight into real world challenges relating to implementation.

Interested schools will be categorised into tertiles based on the Income Deprivation Affecting Children Index ${ }^{28}$ and further stratified by geographic location, identified by the Edubase database, ${ }^{29}$ and school size according to the number of pupils on roll (from Ofsted Data dashboard ${ }^{\mathrm{iii}}$ ). A minimum of two schools will be selected from each tertile and invited to participate. Where possible, this will include a spread of schools in terms of size and geographic location (urban/rural). Consideration will also be given to each school's capacity to engage with the project based on supplementary insight provided by the Alliance.

Given that this study will explore the school level and individual level characteristics associated with implementation, participants from each school will include the head teachers, year 5 pupils (aged 9-10 years), year 5 class teachers and the Marathon Champion, that is, the member of school staff who takes responsibility for coordinating MK in the school (if not the year 5 teacher). Year 5 , the penultimate year of primary school education was chosen for pragmatic reasons. First, pupils needed to have adequate comprehension for the chosen measures deemed to be valid and reliable, and second to ensure availability for follow-up data collection the subsequent year.

\section{Patient and public involvement}

The teaching school alliance will be consulted on the research design to offer pragmatic solutions to minimise potential burden on teachers and pupils and to ensure that the research will contribute to the support provided to the schools by the alliance for school improvement. In addition, a joint dissemination event with the alliance will be planned to communicate the findings of the research and recommendations for practice to the research schools and others in the Alliance implementing physical activity programmes.

\section{Programme theory}

Realist approaches to evaluation posit that all programmes, regardless of whether they are based on academic

\footnotetext{
ii Personal communication with management staff at the Teaching School Alliance.

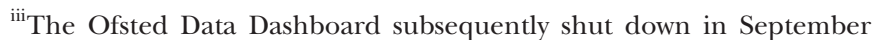
2016.
} 


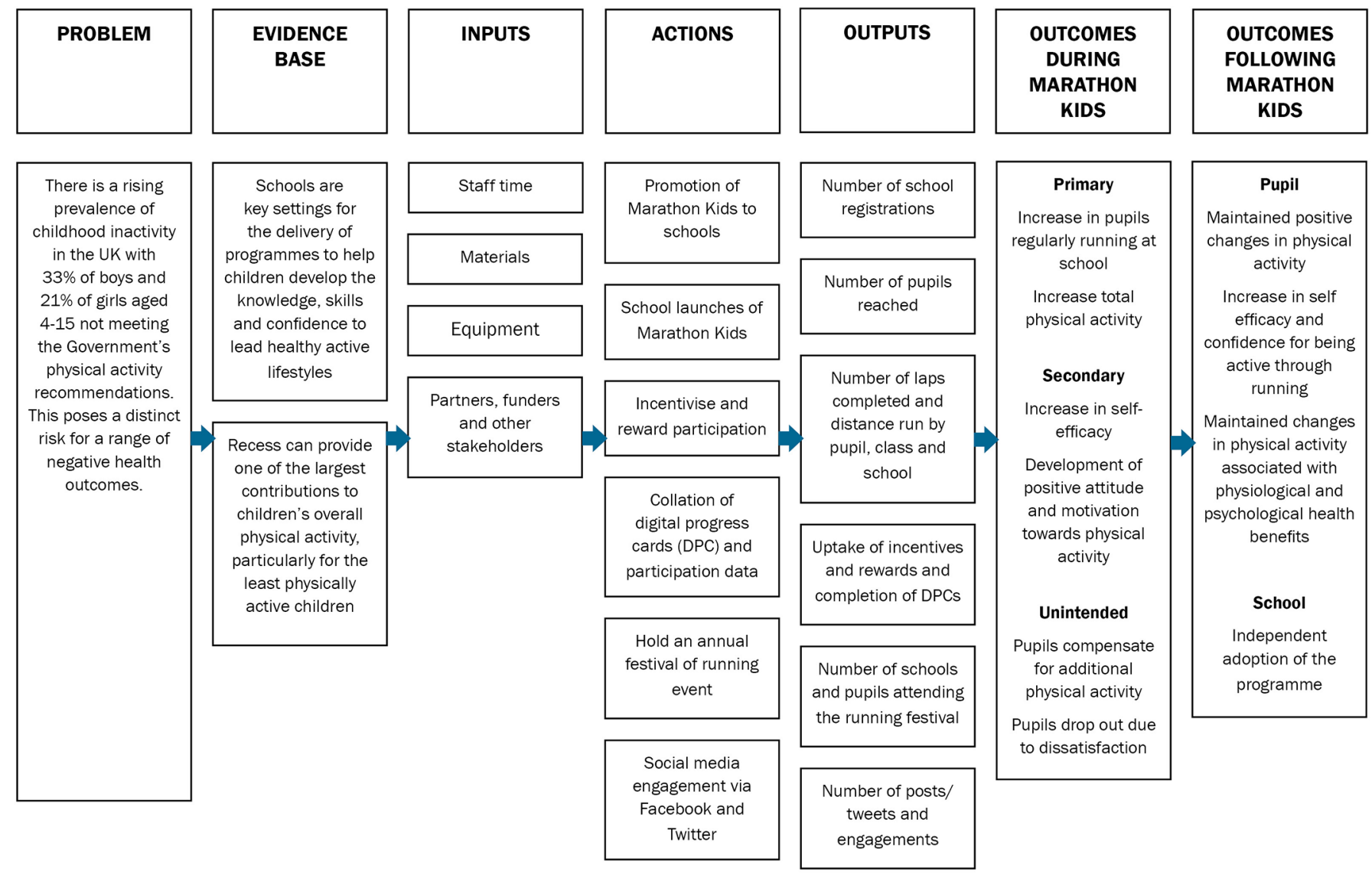

Figure 1 Marathon Kids logic model.

psychology theory, are 'theories incarnate', ${ }^{18}$ that is, whenever a programme is implemented, it is reflecting assumptions about what might cause the problem and how programme components may facilitate change. Identifying these assumptions or 'programme theory' is essential to gain sufficient depth of understanding of the implementation and functioning of the programme and to inform evaluation design. ${ }^{25}$ Thus, interviews and focus groups were conducted, and field notes were recorded in a sample of 20 schools that had implemented MK between 2013 and 2016 (for a detailed description of data collection methods, see Chalkley et al submitted). Data were used to gain information on the programme theory of MK and to inform a programme logic model (see figure 1). The use of logic models in physical activity evaluation has been advocated to improve their quality ${ }^{30}$ by making explicit the intended core components of the intervention, how they interact to produce change, anticipated short-term, medium-term and long-term outcomes as well as the resources and structures in place to ensure implementation.

\section{Theoretical framework of the implementation evaluation}

Applying theories and methods of implementation research helps evaluators clarify the challenges of implementing programmes such as MK; however, school-based public health implementation research is still in its infancy with much of the literature lacking an explicitly identified theoretical framework. ${ }^{31}$ This evaluation will address key dimensions and theoretical constructs of implementation within widely used frameworks, specifically: reach (rate of involvement and representativeness of pupils participating in MK), fidelity of delivery (extent to which the delivered programme relates to the originally intended programme), dose received (how much of MK has been delivered), implementation quality (how well MK has been delivered by teachers), participant responsiveness (degree to which MK stimulates interest in pupils), adaptation (changes made to MK during implementation) and sustainability (the extent to which MK is maintained or embedded within the schools' usual practice).$^{32}$

\section{Data collection}

Data collection for the evaluation will take place throughout the 2016/2017 academic year with follow-up measures planned for the 2017/2018 academic year (October 2017 and March and June 2018) (see figure 2). During 2016/2017, data will be collected on six occasions. Measurements will be collected at time point (TP) 1 in September 2016, prior to MK being launched in schools. Observation of participation in MK will take place at TP2, TP3 and TP5 (January, March and June 2017). Distance ran by pupils will be objectively measured using a radiofrequency identification (RFID) chip timing system at TP4 (April 2017). Teacher interviews and pupil focus groups will take place at TP2 and TP5 (January and June 


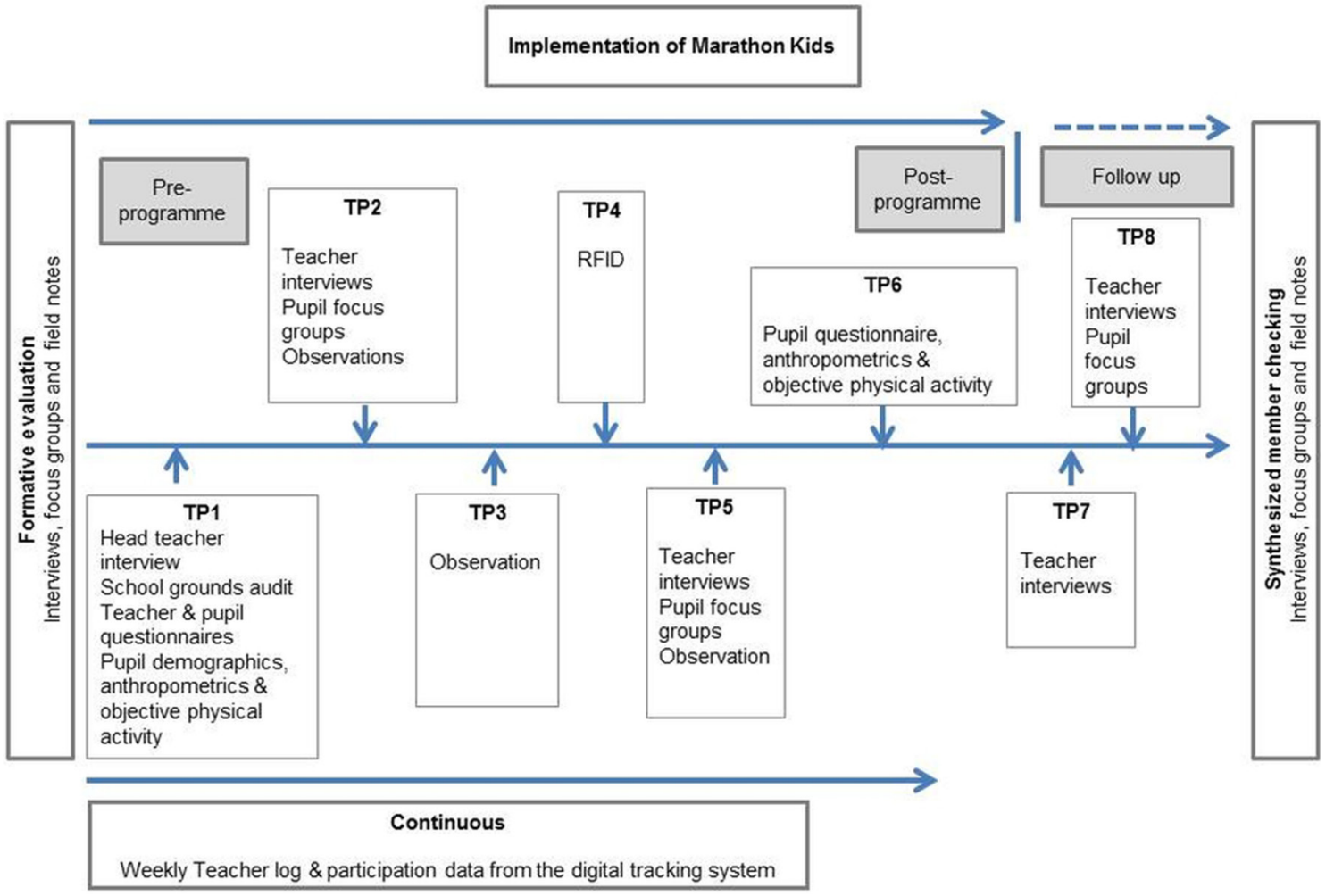

Figure 2 Timeplan for the evaluation of Marathon Kids. RFID, radiofrequency identification; TP, time point.

2017). Measurements will be collected at the end of the 2017/2018 academic year at T6 (July 2017), and follow-up teacher interviews will be conducted at TP7 and TP8 (October 2017 and March 2018) and a follow-up focus group with pupils will be completed at TP8 (March 2018). During the continuation of the $2016 / 2017$ academic year, teachers will also complete an online implementation $\log$, and data from the DTS will be used to verify and/or explain the findings from the implementation evaluation. In addition, validation of the findings will be sought using synthesised member checking. ${ }^{33}$

\section{Measures and instruments}

A number of different measurements and instruments will be used to collect data; these are detailed below according to the level of influence on implementation they will be used to measure.

\section{School level measures \\ School environment}

A number of instruments will be used to collect data pertaining to the school environment, which will be collected at TP1 only (September 2016). First, the paperbased Sport, Physical activity and Eating behaviour: Environmental Determinants in Young people (SPEEDY) Grounds Audit tool ${ }^{34}$ will be completed by the researcher during the initial visit to the school to objectively assess opportunities for physical activity within the school environment.

Second, all year 5 teachers will be asked to complete a paper-based school characteristics questionnaire, relating to their respective school's availability, opportunities and access to physical activity and recreation facilities; physical activity, PE and sport policies and practices (eg, wet weather); the structure of PE classes and clubs; participation in physical activity or sport initiatives or programmes; and accreditation schemes. Questions are adapted from the International Study of Childhood Obesity, Lifestyle and the Environment (ISCOLE) school administrator questionnaire. ${ }^{35}$

Third, pupils' perceptions of the school physical activity environment will be assessed using a modified version of the paper-based Questionnaire Assessing School Physical Activity Environment (Q-SPACE-R) ${ }^{36}$ The Q-SPACE-R has 16 items: eight items assess a school's physical, physical activity environment (ie, equipment, facility quality and programming) and eight items assess a school's social physical activity environment.

Finally, the head teacher in each of the schools will be asked to participate in a semistructured interview to elicit contextual information including the school's policies, practices and ethos relating to the promotion, teaching and delivery of physical activity and healthy lifestyles, as well as of the influences on their decision to adopt the programme.

Individual level measures

\section{Teachers}

Demographic, teaching qualifications, experience and physical activity level: at TP1, teachers will be asked to complete a series of selected response and open-ended questions to 
report their teaching experience, qualifications, professional development relating to sport, $\mathrm{PE}$, physical activity and health. In addition, teachers will be asked to self-report their level of physical activity using a single-item physical activity measure. ${ }^{37}$

\section{Semistructured interviews}

All year five teachers and MK Champions (if different) will participate in semistructured face-to-face interviews. At TP2 and TP5, interviews will be used to elicit information on characteristics of pupils and schools (participating and non-participating); the extent to which components of the intervention were completed and used; the delivery of MK and teacher satisfaction; pupil behaviour; uptake by pupils; and perceptions of influences on uptake. At TP7 and TP8, questions will explore the long-term effects on pupils, teachers and school(s); changes to policy and practice; and any factors affecting sustainability.

\section{Teacher log}

To obtain data on the frequency and duration of exposure to MK as well as uptake, teachers will be asked to complete a weekly log of MK activities during the implementation period (October 2016-June 2017). Teachers will receive an email prompt with a link to an online survey consisting of nine questions that will elicit data on each class' participation in the intervention. In addition to recording frequency and duration of use of the intervention during the week, five items will collect the following information on each occasion: the surface/route; supervisor overseeing participation; any disruptions to lessons as a result of participation; teacher satisfaction; plus a free text box for teachers to provide thoughts and reflections on programme delivery and add any contextual information on factors affecting implementation that week (eg, a special event, teacher absence, examinations and weather).

\section{Pupils}

\section{Demographics}

At TP1, each pupil's name, date of birth, ethnicity, free school meal status and home postcode will be collected from the schools' management information systems. Home postcode will be used to calculate area level socioeconomic status using the 2015 Index of Multiple Deprivation (IMD).$^{28}$

\section{Anthropometrics}

At TP1 and TP6, pupils' standing height will be measured in school by trained researchers using a standard protocol. Measurements will be taken to the nearest $0.1 \mathrm{~cm}$ using a free-standing portable stadiometer (Seca, Seca, Leicester, UK). Height will be measured twice, with a third observation taken if the values differ by greater than $0.4 \mathrm{~cm}$. Weight will be measured to the nearest $0.1 \mathrm{~kg}$ using digital weighing scales (Seca 899, Seca). Body mass index (BMI) will be calculated from height and body mass as a proxy measure of body composition $\left(\mathrm{kg} \cdot \mathrm{m}^{2}\right)$ and BMI z-scores assigned to each pupil. Age-specific and sex-specific cut points will be used to classify children's weight status based on UK reference data. ${ }^{38}$

\section{Objective physical activity}

At TP1 and TP6, pupils will be asked to wear an Actigraph accelerometer (GT3X, GT3X+ or BT) over the right hip using an elasticated waist band during all waking hours for seven consecutive days. These devices have been identified as having acceptable validity and reliability in children ${ }^{39}$ and have been successfully used in other school-based physical activity interventions. ${ }^{40}$

Data will be recorded as accelerometer counts and averaged across a $15 \mathrm{~s}$ interval (epoch). Raw Actigraph data files will be reprocessed to derive outcome variables, using custom data reduction software (Kine Soft, V.3.3.67, Loughborough, UK). Non-wear time will be defined as $60 \mathrm{~min}$ of consecutive zero counts, allowing for $2 \mathrm{~min}$ of non-zero interruptions. ${ }^{41}$

The following variables will be derived from the accelerometer data using the Evenson intensity cut-points ${ }^{42}$ :

1. Mean total volume of activity per day (counts per wear minute; school day, total day, and weekday and weekend day).

2. Mean minutes of moderate to vigourous physical activity (MVPA) (school day, total day and weekday and weekend day).

3. Mean minutes of sedentary time (school day, total day and weekday and weekend day).

4. Proportion of children meeting the physical activity guidelines of at least $60 \mathrm{~min}$ of MVPA per day. ${ }^{43}$

\section{Pupil questionnaire}

At TP1 and TP6, pupils will be asked to complete a paperbased questionnaire that will be used to capture the following:

\section{Knowledge and understanding of physical activity and health}

Four questions will assess their knowledge of physical activity as well as their understanding of how physical activity contributes to health. These include: stating how much physical activity young people of their age should do each day in minutes; identifying activities (from a list provided) that would contribute to their daily total; identifying other activities that might contribute towards a young person's daily amount of physical activity; and answering a series of six true or false statements relating to physical activity and health.

\section{Physical activity}

Self-reported physical activity will be measured using the Physical Activity Questionnaire for Children, ${ }^{44}$ a 7 -day recall instrument used to assess general physical activity levels during the school year.

\section{Self-reported physical fitness and sportiness}

Pupils will self-report perceptions of their physical fitness using the International Fitness Scale. ${ }^{45}$ In addition, a single-item question will be used to assess the degree to which pupils perceive themselves to be 'sporty'. 
Participants will be asked to rate themselves on a scale of $0-10$, where $0=$ 'not sporty at all' and $10=$ 'extremely sporty'.

\section{Psychosocial measures}

Several psychosocial measures will be employed with the pupils. Self-efficacy will be assessed using the Physical Activity Self Efficacy Scale. ${ }^{46}$ Enjoyment will be assessed using the Physical Activity Enjoyment Scale. ${ }^{47}$ Global selfworth (six items) and athletic competence (six items) will be assessed using 12 items from the Physical Self Perception Profile for Children. ${ }^{48}$ Attitude towards being active will be assessed using a 14-item questionnaire. ${ }^{49}$ Social support will be assessed using the Social Support for Physical Activity Scale. ${ }^{50}$ Intention to be physically active will be assessed using three items asking about intentions to be active for at least $60 \mathrm{~min}$ every day during the next month adapted from Hagger $e t a l^{51}$ Motivation to be physically active will be measured using the Behavioural Regulation in Exercise Questionnaire. ${ }^{52}$ These instruments were chosen based on evidence of their psychometric properties and suitability given the age and cognitive abilities of this age group. ${ }^{53-55}$

\section{Pupil focus group}

At TP2, TP5, TP7 and TP8, a sample of year 5 pupils from each school will participate in a semistructured focus group. One mixed sex group of six to eight pupils per class will participate and will be selected by their teacher. Guidelines will be provided to the teachers when selecting pupils, such as ensuring a range in relation to age, sex, enthusiasm and participation level in the programme (eg, high, medium and low), as well as in the pupil's ability to communicate their experiences and opinions. Where possible, the same pupils from each school will participate in the focus groups at all time points.

At TP2 and TP5, questions will explore pupils' exposure to, and level of engagement with, the programme as well as perceptions of enjoyment and acceptability. At TP7 and TP8, questions will explore their overall physical activity levels, participation status, perceived impact of MK and barriers and facilitators to participation in MK. Focus groups will be conducted on the school site and at a time deemed by the teachers to be the least disruptive to the school day.

\section{Running programme participation}

As participation in physical activity is complex, ${ }^{56}$ detailed information will be triangulated via a number of different sources.

\section{Digital tracking system}

During the intervention period (October 2016-June 2017), data from the schools' DTS will be collected every half term via KRF. This contains a record of how far each individual child has run based on the school administered lap band system. Data will be verified using contextual information from the teacher log.

\section{Objective measurement of distance}

At TP4, distance and speed, during a one-off running session, will be objectively measured using the The Orbiter RFID chip timing system. Data will be used to provide an indication of pupils' intensity of activity while participating in MK.

\section{Direct observation of physical activity}

At TP2, TP3 and TP5, pupils' use of the programme during a lunchtime period will be observed. Uptake will be assessed using an observation protocol based on the Children's Activity Rating Scale (CARS) ${ }^{57}$ in order to code physical activity behaviour. CARS defines observed activities into five intensity categories: stationary, stationary with limb or trunk movements, slow movement, moderate movement and fast movement, and discriminates between levels of energy expenditure in children. An additional coding system will be used to capture the social context of the physical activity behaviour such as pupil and teacher interaction and use of the lap bands.

\section{Field notes}

Field notes will be recorded to capture supplementary data in order to aid understanding of the data captured using the other methods. These will include those taken by the member of staff from KRF following the delivery of the school launch, as well as those from the lead researcher during each data collection visit. Consideration will be given to observations of school context, teacher and pupil engagement and social interactions.

\section{Synthesised member checking}

In order to validate the refined programme theory, synthesised member checking will be used to gain consensus as to the degree it resonates with Marathon Champions, year 5 teachers and year 5 pupils within a mixed sample of schools. Participants will be recruited from schools that will be implementing MK for the first time during the $2017 / 2018$ academic year as well as from some of the 20 schools that participated in the development of the initial programme theory. Interviews (Marathon Champions and/or year 5 teacher) and focus groups (year 5 pupils) will be conducted (June 2018) to explore the degree to which the refined programme theory is reflective of their experiences. Member checking is an appropriate method to employ within the realist evaluation framework, particularly where data have been collected from different sources and/or at different time points ${ }^{58}$ and will limit the potential for researcher bias by actively engaging participants in checking and confirming the results.

\section{ANALYSES OF DATA}

Analysis of the qualitative data will be concurrent with data collection to allow the identification of further hypothetical pathways and allow emergent lines of enquiry to be explored. All interviews and focus groups will be audio-recorded and transcribed verbatim into Microsoft Word 
before being imported into NVivo 10 software. Consistent with realist evaluation methodologies, thematic analysis of the interviews will be guided by the initial programme theories identified in the formative evaluation (Chalkley et al submitted). The developed theory of context, mechanism and outcome will be tested using deductive coding; however, analysis will be open to emergent themes based on the principle of inductive content analysis to facilitate further theory refinement. ${ }^{59}$

Descriptive analysis will be undertaken to describe the school (eg, school-level deprivation), teacher (eg, number of years of teaching experience) and pupil level (eg, ethnicity) baseline characteristics, as well as level of implementation (obtained from teacher logs) over time and also between schools. Appropriate summary measures including totals, percentages, means and standard deviations, medians and interquartile ranges will be used.

If appropriate, a linear multilevel model will be used to examine whether the level of implementation is associated with participation. This will include participation data (from the DTS) as the outcome variable, levels to indicate the clustering of pupils within a class and within schools and a categorical indicator for level of implementation (from the teacher logs) as the explanatory variable. Where there is insufficient data to undertake a regression analysis, exploratory analysis will be undertaken using t-tests to consider how mechanisms produce outcomes in an accompanying context, as per the realist evaluation methodology. To examine factors associated with implementation, potential mediating (eg, self-efficacy), moderating (eg, social support) and a priori confounder variables (eg, IMD) will be included.

The combination of both qualitative and quantitative data will allow the verification of data using triangulation. ${ }^{60}$ Based on a convergent parallel design, qualitative and quantitative data will be collected in parallel, analysed separately and then merged. This will provide a more holistic understanding of the implementation of MK on both the individual and school levels.

A matrix will be used to analyse and synthesise both the qualitative and quantitative data available for each school. The columns of the matrix will contain the individual CMO configurations, and each row in the matrix will represent a different school. This approach will be used to facilitate within-case analysis highlighting similarities and discrepancies between data sources as well as cross-case analysis to identify patterns across cases. This will inform the acceptance, modification or rejection of the CMO hypotheses to inform a plausible explanation of if, how and why MK is effective.

\section{DISCUSSION}

The combination of political interest ${ }^{61}$ increased investment $^{45}$ and public health necessity ${ }^{3}$ have resulted in school-based physical activity programmes receiving much attention. ${ }^{62}$ However, despite the numerous research studies on what works to promote and increase school-based physical activity, there is a lack of generalisable, effective and sustainable programmes that have been translated into practice. ${ }^{63}$

This study will provide a timely examination of the implementation of a school-based running programme, helping to contribute to our understanding of implementing running specific programmes and school-based physical activity programmes more broadly. Understanding the ways in which head teachers, school staff and pupils interact with and perceive a programme is essential for determining how and why it is or is not effective.

This paper has outlined the protocol for a mixed methods study to investigate the implementation of a school-based running programme in UK primary schools and establish which aspects of the programme are working, for whom and in which circumstances. Data from the implementation evaluation will be used to unpack the processes of engagement and participation, which are hypothesised to mediate the programme's success. Use of the realist approach will help to identify and test the hypothesised causal mechanisms, evaluate the extent to which MK activated them, use this analysis to refine the programme theory and identify areas of strength and potential future improvement in the programme design. The identification of underlying causal mechanisms and the development of propositions will enhance the utility and transferability of the findings. ${ }^{22}$

Given the popularity of school-based running programmes and the growing awareness of programmes among the general public, teachers and policy makers, the findings of this study will be of great interest and relevance and will help to inform national strategies to support quality implementation. Furthermore, findings will contribute more generally to our understanding of implementing physical activity programmes within the complex educational setting of schools.

\section{STRENGTHS AND LIMITATIONS}

The strengths of this study include: the recruitment strategy to ensure a representative sample (in terms of size, socioeconomic status and geographic location), the use of mixed methods and measures at multiple levels to provide a more holistic understanding of the implementation, plus the adoption of a realist framework, which encourages exploration of the complexity of implementing programmes in a school setting. Due to financial limitations and the ambition to have equitable representation of one age group, the evaluation only focuses on 1 year group, which may limit the applicability of the findings across the primary school setting more broadly.

\section{ETHICS AND DISSEMINATION}

Informed written consent has been sought from each participant prior to conducting the interviews, and all pupils will be asked to assent prior to their participation in data collection. Parental consent to participate in evaluation activities has also been sought from pupil's 
parents/carers. All participants will be given a unique identification number, and anonymity will be assured at each school site.

The study results will be embedded in the development of the programme to contribute to future decision making and inform an evidence-based strategic approach to improve implementation quality and effectiveness. Dissemination will be targeted at multiple levels and will include: a project research report for the funder, circulation of findings to public health practitioners via the opportunities available through the National Centre for Sport and Exercise Medicine, via publications in peer-reviewed journals and attendance at conferences and events for those working within the field of physical activity and public health.

Acknowledgements We are very grateful to the pupils, teachers, schools and Kids Run Free staff who contributed to the formative piece of work that informed the study design as well as the Teaching School Alliance for their support with this research.

Contributors AEC, ACR and LBS initially conceived the original study design, and $\mathrm{LAC}, \mathrm{JPH}$ and TG helped refine the study design. AEC wrote the first draft of the manuscript. ACR, JPH, LAC, TG and LBS reviewed and contributed feedback and critical comments on drafts of the manuscript before approving the final version.

Funding This study was funded by London Marathon Events Ltd and Kids Run Free. This work was supported by the National Institute for Health Research Collaboration for Leadership in Applied Health Research and Care - East Midlands (NIHR CLAHRC-EM) and the National Institute for Health Research (NIHR) Leicester Biomedical Research Centre.

Disclaimer The views expressed in this publication are those of the authors and not necessarily those of London Marathon Events Ltd and Kids Run Free, the NHS, the National Institute for Health Research or the Department of Health.

Competing interests None declared.

Patient consent Not required.

Ethics approval The study has been approved by the Human Participants Ethics Subcommittee of Loughborough University (R16-P032 and R16-P116).

Provenance and peer review Not commissioned; externally peer reviewed.

Open Access This is an Open Access article distributed in accordance with the Creative Commons Attribution Non Commercial (CC BY-NC 4.0) license, which permits others to distribute, remix, adapt, build upon this work non-commercially, and license their derivative works on different terms, provided the original work is properly cited and the use is non-commercial. See: http://creativecommons.org/ licenses/by-nc/4.0/

(c) Article author(s) (or their employer(s) unless otherwise stated in the text of the article) 2018. All rights reserved. No commercial use is permitted unless otherwise expressly granted.

\section{REFERENCES}

1. The Lancet. Ready, set, go for physical activity. Lancet 1960;2013:381.

2. The Lancet. UK Government won't step up to the plate on childhood obesity. Lancet 2016;388:841

3. Department of Health. Childhood Obesity: A Plan for Action, 2016.

4. Department for Culture Media \& Sport, Department for Education. 2010 to 2015 government policy: sports participation - Publications - GOV.UK. 2013 https://www.gov.uk/government/publications/2010to-2015-government-policy-sports-participation.

5. Department for Education. New fudning to boost schools facilities and healthy lifestyles. 2017 https://www.gov.uk/government/news/ new-funding-to-boost-schools-facilities-and-healthy-lifestyles (accessed 20 Oct 2017).

6. The Daily Mile. www.thedailymile.co.uk (accessed 23 Jan 2018).
7. National Centre for Sport and Medicine. Making Strides in Schools symposium report. 2016 http://www.ncsem-em.org.uk/makingstrides-in-schools/.

8. Naylor PJ, McKay HA. Prevention in the first place: schools a setting for action on physical inactivity. Br J Sports Med 2009;43:10-13.

9. Dane AV, Schneider BH. Program integrity in primary and early secondary prevention: are implementation effects out of control? Clin Psychol Rev 1998;18:23-45.

10. Durlak JA. Why Program Implementation is Important. J Prev Interv Community 1998;17:5-18.

11. Dobbins M, Husson H, DeCorby K, et al. School-based physical activity programs for promoting physical activity and fitness in children and adolescents aged 6 to 18. Cochrane Database Syst Rev 2013;2:CD007651.

12. Borde R, Smith JJ, Sutherland R, et al. Methodological considerations and impact of school-based interventions on objectively measured physical activity in adolescents: a systematic review and meta-analysis. Obes Rev 2017;18:476-90.

13. Moore GF, Audrey S, Barker M, et al. Process evaluation of complex interventions: Medical Research Council guidance. BMJ 2015;350:h1258.

14. Fletcher A, Jamal F, Moore G, et al. Realist complex intervention science: Applying realist principles across all phases of the Medical Research Council framework for developing and evaluating complex interventions. Evaluation 2016;22:286-303.

15. Rowling L, Samdal O. Filling the black box of implementation for health-promoting schools. Health Educ 2011;111:347-62.

16. Christian D, Todd C, Davies $\mathrm{H}$, et al. Community led active schools programme (CLASP) exploring the implementation of health interventions in primary schools: headteachers' perspectives. BMC Public Health 2015;15:238.

17. Jago R, Rawlins E, Kipping RR, et al. Lessons learned from the AFLY5 RCT process evaluation: implications for the design of physical activity and nutrition interventions in schools. BMC Public Health 2015;15:946.

18. Lendrum A, Humphrey N. The importance of studying the implementation of interventions in school settings. Oxf Rev Educ 2012;38:635-52.

19. Marathon Kids. www.marathonkids.org.uk (accessed 23 Jan 2018)

20. Golden Mile. http://www.golden-mile.org/ (accessed 4 Jun 2016).

21. Springer AE, Kelder SH, Ranjit N, et al. Promoting physical activity and fruit and vegetable consumption through a communityschool partnership: the effects of Marathon Kids ${ }^{\circledR}$ on lowincome elementary school children in Texas. J Phys Act Health 2012;9:739-53.

22. Pawson R, Tilley N. Realistic evaluation: Sage, 1997.

23. Pawson R, Manzano-Santaella A. A realist diagnostic workshop. Evaluation 2012;18:176-91

24. Merton R. Social Theory and Social Structure. New York: Free Press, 1968.

25. Pawson $\mathrm{R}$. The science of evaluation : a realist manifesto: SAGE, 2013.

26. Palinkas LA, Aarons GA, Horwitz S, et al. Mixed Method Designs in Implementation Research. Administration and Policy in Mental Health and Mental Health Services Research 2011;38:44-53.

27. Southam-Gerow MA, Dorsey S. Qualitative and mixed methods research in dissemination and implementation science: introduction to the special issue. J Clin Child Adolesc Psychol 2014;43:845-50.

28. Department for Communities and Local Government. Index of Deprivation Affecting Children Index, 2015.

29. Edubase. www.education.gov.uk/edubase/home.xhtml (accessed 4 May 2016).

30. Cavill N, Roberts K, Rutter H. Standard Evaluation Framework for physical activity interventions. Public Health 201238 https://www. noo.org.uk/core/frameworks/SEF PA

31. Lee R, Gortmaker S. Health dissemination and implementation within schools. In: Brownson RC, Colditz GA, Proctor EK, eds. Dissemination and Implementation Research in Healthcare. Oxford University Press, 2012:419-37.

32. Durlak JA, DuPre EP. Implementation matters: a review of research on the influence of implementation on program outcomes and the factors affecting implementation. Am J Community Psychol 2008;41:327-50.

33. Birt L, Scott S, Cavers D, et al. Member Checking. Qual Health Res 2016;26:1802-11.

34. Jones NR, Jones A, van Sluijs EM, et al. School environments and physical activity: The development and testing of an audit tool. Health Place 2010;16:776-83.

35. Katzmarzyk PT, Barreira TV, Broyles ST, et al. The International Study of Childhood Obesity, Lifestyle and the Environment (ISCOLE): design and methods. BMC Public Health 2013;13:900. 
36. Martin JJ, McCaughtry N, Flory S, et al. Validity and Reliability of the School Physical Activity Environment Questionnaire. Meas Phys Educ Exerc Sci 2011;15:274-82.

37. Milton K, Bull FC, Bauman A. Reliability and validity testing of a single-item physical activity measure. Br J Sports $\mathrm{Med}$ 2011;45:203-8.

38. Cole TJ, Freeman JV, Preece MA. Body mass index reference curves for the UK, 1990. Arch Dis Child 1995;73:25-9.

39. Hänggi JM, Phillips LR, Rowlands AV. Validation of the GT3X ActiGraph in children and comparison with the GT1M ActiGraph. $J$ Sci Med Sport 2013;16:40-4.

40. Sutherland R, Campbell E, Lubans DR, et al. A cluster randomised trial of a school-based intervention to prevent decline in adolescent physical activity levels: study protocol for the 'Physical Activity 4 Everyone' trial. BMC Public Health 2013;13:57.

41. Troiano RP, Berrigan D, Dodd KW, et al. Physical activity in the United States measured by accelerometer. Med Sci Sports Exerc 2008; $40: 181-8$

42. Evenson KR, Catellier DJ, Gill K, et al. Calibration of two objective measures of physical activity for children. J Sports Sci 2008;26:1557-65.

43. Department of Health. Start Active, Stay Active. Report, 2011:62.

44. Kowalski KC, Crocker PRE, Faulkner RA. Validation of the Physical Activity Questionnaire for Older Children. Pediatr Exerc Sci 1997;9:174-86.

45. Ortega FB, Ruiz JR, España-Romero V, et al. The International Fitness Scale (IFIS): usefulness of self-reported fitness in youth. Int $J$ Epidemiol 2011;40:701-11.

46. Saunders RP, Pate RR, Felton G, et al. Development of Questionnaires to Measure Psychosocial Influences on Children's Physical Activity. Prev Med 1997;26:241-7.

47. Kendzierski D, DeCarlo KJ. Physical Activity Enjoyment Scale: Two Validation Studies. Journal of Sport and Exercise Psychology 1991;13:50-64.

48. Harter S. The Perceived Competence Scale for Children. Child Dev 1982;53:87-97.

49. Nelson TD, Benson ER, Jensen CD. Negative attitudes toward physical activity: measurement and role in predicting physical activity levels among preadolescents. $J$ Pediatr Psychol 2010;35:89-98.
50. Sallis JF, Grossman RM, Pinski RB, et al. The development of scales to measure social support for diet and exercise behaviors. Prev Med 1987;16:825-36.

51. Hagger M, Chatzisarantis NL, Hein V, et al. Teacher, peer and parent autonomy support in physical education and leisure-time physical activity: A trans-contextual model of motivation in four nations. Psychol Health 2009;24:689-711.

52. Markland D, Tobin V. A Modification to the Behavioural Regulation in Exercise Questionnaire to Include an Assessment of Amotivation. Journal of Sport and Exercise Psychology 2004;26:191-6.

53. Dishman RK, Motl RW, Sallis JF, et al. Self-management strategies mediate self-efficacy and physical activity. Am J Prev Med 2005;29:10-18

54. Moore JB, Yin Z, Hanes J, et al. Measuring Enjoyment of Physical Activity in Children: Validation of the Physical Activity Enjoyment Scale. J Appl Sport Psychol 2009;21:S116-S129.

55. Sebire SJ, Jago R, Fox KR, et al. Testing a self-determination theory model of children's physical activity motivation: a cross-sectional study. Int J Behav Nutr Phys Act 2013;10:111.

56. Sallis JF, Prochaska JJ, Taylor WC. A review of correlates of physical activity of children and adolescents. Med Sci Sports Exerc 2000;32:963-75

57. Finn KJ, Specker B. Comparison of Actiwatch activity monitor and Children's Activity Rating Scale in children. Med Sci Sports Exerc 2000;32:1794-7.

58. Triangulation TH. Respondent Validation, and Democratic Participation in Mixed Methods Research. J Mix Methods Res 2012;6:111-23.

59. Elo $\mathrm{S}$, Kyngäs $\mathrm{H}$. The qualitative content analysis process. J Adv Nurs 2008;62:107-15.

60. Green J, Thorogood N. Qualitative methods for health research. SAGE PublicationsSage UK: London, England, 2013.

61. Scottish Government. Scotland: a Daily Mile Nation. https://news. gov.scot/news/scotland-a-daily-mile-nation (accessed 25 Jan 2018).

62. Inman DD, van Bakergem KM, Larosa AC, et al. Evidence-based health promotion programs for schools and communities. Am J Prev Med 2011:40:207-19.

63. McKay HA, Macdonald HM, Nettlefold L, et al. Action Schools! BC implementation: from efficacy to effectiveness to scale-up. $\mathrm{Br} J$ Sports Med 2015;49:210-8. 